\title{
Identification, cloning and characterization of the tomato TCP transcription factor family
}

\author{
Violeta Parapunova ${ }^{1,2}$, Marco Busscher ${ }^{2}$, Jacqueline Busscher-Lange ${ }^{2}$, Michiel Lammers ${ }^{2}$, Rumyana Karlova ${ }^{2,3}$,
} Arnaud G Bovy ${ }^{2}$, Gerco C Angenent ${ }^{1,2}$ and Ruud A de Maagd ${ }^{2^{*}}$

\begin{abstract}
Background: TCP proteins are plant-specific transcription factors, which are known to have a wide range of functions in different plant species such as in leaf development, flower symmetry, shoot branching, and senescence. Only a small number of TCP genes has been characterised from tomato (Solanum lycopersicum). Here we report several functional features of the members of the entire family present in the tomato genome.

Results: We have identified 30 Solanum lycopersicum SITCP genes, most of which have not been described before. Phylogenetic analysis clearly distinguishes two homology classes of the SITCP transcription factor family - class I and class II. Class II differentiates in two subclasses, the CIN-TCP subclass and the CYC/TB1 subclass, involved in leaf development and axillary shoots formation, respectively. The expression patterns of all members were determined by quantitative PCR. Several SITCP genes, like SITCP12, SITCP15 and SITCP18 are preferentially expressed in the tomato fruit, suggesting a role during fruit development or ripening. These genes are regulated by RIN (RIPENING INHIBITOR), CNR (COLORLESS NON-RIPENING) and SIAP2a (APETALA2a) proteins, which are transcription factors with key roles in ripening. With a yeast one-hybrid assay we demonstrated that RIN binds the promoter fragments of SITCP12, SITCP15 and SITCP18, and that CNR binds the SITCP18 promoter. This data strongly suggests that these class I SITCP proteins are involved in ripening. Furthermore, we demonstrate that SITCPs bind the promoter fragments of members of their own family, indicating that they regulate each other. Additional yeast one-hybrid studies performed with Arabidopsis transcription factors revealed binding of the promoter fragments by proteins involved in the ethylene signal transduction pathway, contributing to the idea that these SITCP genes are involved in the ripening process. Yeast two-hybrid data shows that SITCP proteins can form homo and heterodimers, suggesting that they act together in order to form functional protein complexes and together regulate developmental processes in tomato.

Conclusions: The comprehensive analysis we performed, like phylogenetic analysis, expression studies, identification of the upstream regulators and the dimerization specificity of the tomato TCP transcription factor family provides the basis for functional studies to reveal the role of this family in tomato development.
\end{abstract}

Keywords: Transcription factors, Tomato, Yeast one-hybrid, Yeast two-hybrid

\section{Background}

TCP proteins, named after the three first characterized family members TEOSINTE BRANCHED (TB) 1 from maize, CYCLOIDEA (CYC) from Antirrhinum majus, and PROLIFERATING CELL FACTORS (PCFs) from rice are plant-specific transcription factors characterized by the TCP domain, a motif encompassing a non-canonical basic-helix-loop-helix (bHLH) structure [1]. While initially

\footnotetext{
* Correspondence: ruud.demaagd@wur.nl

${ }^{2}$ Plant Research International, P.O. Box 619, 6700 AP Wageningen, the Netherlands

Full list of author information is available at the end of the article
}

these transcription factors were implicated in the regulation of growth and development, it has become apparent that they are involved in many processes including senescence, circadian rhythm and hormone signaling (reviewed in: $[2,3])$. Based on the homology of the TCP domains, TCP proteins can be divided into two major classes, class I and class II. The first is represented by the rice PCF proteins and many of its members have no known function so far. The Arabidopsis gene AtTCP14 has been shown to regulate embryonic growth potential in Arabidopsis seeds [4] and together with AtTCP15, it regulates internode length [5]. AtTCP20 appears to function in diverse developmental 
processes, such as growth processes [6], jasmonic acid (JA) biosynthesis and leaf senescence [7]. AtTCP16 is predominantly expressed in developing microspores, and its down-regulation in transgenic plants resulted in $50 \%$ abnormal pollen [8].

Class II, represented by $C Y C$ and $T B 1$, contains most genes with known functions. The $C Y C$ gene, together with the related DICHOTOMA $(D I C H)$ in Antirrhinum is required for dorsoventral asymmetry of the flower [9]. The Arabidopsis CYC/DICH homolog AtTCP1 regulates the expression of the brassinosteroid synthetic gene DWARF4 and is thus also linked to growth [10]. The TB1 gene affects the fate of maize axillary meristems. It prevents the outgrowth of buds at the lower nodes and it promotes the formation of female inflorescences at the higher nodes [11]. In Arabidopsis, two homologs of TB1, BRANCHED1 (BRC1, AtTCP18) and BRANCHED2 (BRC2, AtTCP12) are expressed in axillary buds, and mutants with reduced activity of either gene show increased branching [12]. The tomato orthologs SIBRC1a and SlBRC1b have similar functions in tomato axillary bud initiation and outgrowth [13].

Other examples of TCP genes affecting plant architecture are CINCINATTA in Antirrhinum [14] and its homolog LANCEOLATE (LA, SlTCP2) in tomato [15]. The dominant Lanceolate mutation in tomato produces small simple leaves instead of the normally large and compound ones. The corresponding TCP gene allele la contains point mutations in a miR319-binding site, leading to reduced sensitivity to miRNA regulation. One other full-length mRNA and two mRNA fragments with putative miR319binding sites, designated SlTCP3, SlTCP10 and SITCP24 after their closest homologs in Arabidopsis, respectively, were also identified [15]. The Arabidopsis homologs, as well as AtTCP2 and AtTCP4 (the closest homologs of $L A N C E O L A T E$ and $C I N$ ) have been earlier identified as targets of miRNA319 through activation-tagging mutants of the miRNA319-encoding $J A W$ locus [16] and are therefore, called $J A W$ clade TCP genes. In the activation-tagged jawD mutant, which exhibits a reduced expression of the $J A W$ clade $T C P$ genes, the differential regulation of cell division during leaf development is disturbed, causing negative leaf curvature and crinkly leaves [17,18]. MiRNA319-targetting AtTCP4 is required for petal growth and development [19]. The same clade of miRNA319-regulated genes in Arabidopsis has been shown to control JA synthesis and leaf senescence [20]. Studies in Arabidopsis suggest that CCA1 HIKING EXPEDITION (CHE) (AtTCP21) is involved in circadian clock regulation by repressing the CIRCADIAN CLOCK ASSOCIATED 1 (CCA1) gene [21].

A group of homologous TCPs of Arabidopsis functions redundantly in the control of shoot lateral organ morphology through the negative regulation of boundary-specific genes such as CUP-SHAPED COTELYDON 1 [22]. The only known example of a TCP gene affecting fruit development is the phenotype of a dominant-negative variant of AtTCP3, where the siliques are shorter and their surface wrinkled [22].

As in other transcription factors, the basic region of the TCP domain is likely to be involved in DNA binding, but deletion studies have shown that both $\mathrm{N}$ - as well as $\mathrm{C}$-terminal regions of the conserved bHLH-domain are required for target site recognition by the rice PCF1 [23]. Modeling of Arabidopsis TCP4 dimers bound to target DNA suggest that the first part of the basic region of the TCP domain forms a small $\alpha$-helix involved in DNA base interactions and the C-terminal part of this region may form an $\alpha$-helix contiguous with Helix 1 . Homology modeling based on the animal bHLH protein myoD suggests that the Helix-loop-helix region is responsible for dimerization [24]. It was shown that TCP proteins tend to form homodimers or heterodimers with other TCP proteins of the same class [25].

The consensus binding site sequences for the two classes are distinct, but overlapping (GGNCCCAC for class I and GTGGNCCC for class II) and the core (GGNCCC) shared by these sequences has a strict role in the binding of both classes [25]. TCP-binding elements are found in the promoters of various cell cycle related genes and of genes encoding ribosomal proteins [26]. AtTCP20 has been found to physically bind to synthetic versions of these elements, as well as to cis-elements in the promoter of the mitotic cyclin $C Y C B 1 ; 1$ gene. It was proposed that organ growth rates and possibly shape are regulated by the balance between positively and negatively acting TCP proteins competing for binding to the same promoters [27]. Later, it was found that class I TCP proteins act antagonistically to the class II $J A W$-TCPs via the JA signaling pathway, as TCP20 inhibits LOX2 (LIPOXYGENASE 2) and TCP4 induces LOX2 expression [7].

In this manuscript we describe the identification and characterization of 30 different TCP-encoding genes from tomato (Solanum lycopersicum). Using quantitative RTPCR we have determined their expression in different tissues and during fruit development, revealing differential expression patterns of members during fruit development and ripening. The latter was shown to be dependent on several major ripening regulatory transcription factors like LeMADS-RIN (RIPENING-INHIBITOR) [28], a MADS box protein, COLORLESS NON-RIPENING (CNR), a SQUAMOSA promoter binding protein (SBP) [29], and APETALA2a (SlAP2a), an APETALA2/ETHYLENE RESPONSE FACTOR (AP2/ERF) [30]. These ripeningassociated transcription factors regulate ripening through the biosynthesis of ethylene and/or its signalling.

This is the first study revealing the correlation of TCP transcription factors in fleshy fruit development and 
ripening. Moreover, we further investigated their regulation by identification of transcription factors interacting with promoter sequences of these genes in a yeast one-hybrid assay. Furthermore, in a yeast 2-hybrid assay we have determined the capacity of the tomato TCP proteins to form homo- and heterodimeric interactions. Comparison of the characteristics of the tomato family members with those from other plant species may reveal common and diverged features and may give clues about the function of the tomato TCP genes.

\section{Results and discussion}

\section{Identification and cloning of tomato TCP genes}

By mining the tomato Unigene and BAC sequence databases from the Sol Genomics Network with homology searches and subsequent sequence extension by RACE, we initially identified and cloned 24 different tomato genes encoding putative TCP transcription factors (Table 1; SlTCP1-24). Many of the sequences we have identified had only 1 or few representative EST in the databases. Four genes had no representative EST and were identified directly from the genomic sequence available at the time. Publication of the tomato genome sequence allowed the identification of another 6 SITCP genes (Table 1). Of the 30 identified unique genes, 2 full-length mRNA sequences, for Lanceolate and SITCP3 [15], and three partial sequences, previously named SlTCP1, SlTCP2, and SlTCP3 [31], here renamed SlTCP22, SlTCP7, and SITCP8, respectively, were already present in Genbank. SITCP7 and SITCP9 [31] were subsequently also named $B R C 1 B$ and $B R C 1 A$, respectively [13].

\section{Genomic organization and phylogenetic analysis}

The chromosome location of the 30 genes is depicted in Additional file 1: Figure S1. We found in the published Heinz 1706 genome (v2.40) four additional full length copies of SlTCP1(named TCP1a-d) and one partial copy each of SITCP19 (a) and SlTCP28 (a), respectively, in different genomic locations (listed in Additional file 2: Table S1 and shown in Additional file 1: Figure S1). The additional copies of SITCP1 have in their close vicinity open reading frames with homology to transposon sequences, suggesting that they are the result of mobilization by transposable element activity. Since our experimentally determined mRNA sequences as well as publicly available EST sequences map uniquely to the respective genes and not to these additional copies, we conclude that these copies are not expressed under conditions used by ourselves or by others. This is further supported by the observation of genomic synteny in the Plant Genome Duplication Database (PGDD) [32], where SlTCP1 and its genomic environment is contained in a block showing extensive synteny with 22 different genomic sequence blocks in 11 plant species, with the four copies (SlTCP1a-d)
Table 1 Tomato TCP genes

\begin{tabular}{|c|c|c|c|}
\hline Name & iTAG2.3 & $\begin{array}{l}\text { Previous/ } \\
\text { alternate name }\end{array}$ & Accession no. \\
\hline SITCP1 & Solyc02g077250.2 & & GQ496320 \\
\hline SITCP2 & Solyc07g062680.1 & Cycloidea/Lanceolate & AF175965 \\
\hline SITCP3 & Solyc12g014140.1 & SITCP3 (fragment) & GQ496321 \\
\hline SITCP4 & Solyc03g115010.1 & & GQ496322 \\
\hline SITCP5 & Solyc02g089020.1 & & GQ496323 \\
\hline SITCP6 & Solyc06g069460.1 & & GQ496324 \\
\hline SITCP7 & Solyc02g089830.1 & SITCP2 (fragment)/BRC1B & GQ496325 \\
\hline SITCP8 & Solyc06g069240.1 & SITCP3 (fragment) & GQ496326 \\
\hline SITCP9 & Solyc03g119770.2 & $B R C 1 A$ & GQ496327 \\
\hline SITCP10 & Solyc07g053410.2 & & GQ496328 \\
\hline SITCP11 & Solyc01g103780.2 & & GQ496329 \\
\hline SITCP12 & Solyc11g020670.1 & & GQ496330 \\
\hline SITCP13 & Solyc06g065190.1 & & GQ496331 \\
\hline SITCP14 & Solyc04g009180.1 & & GQ496332 \\
\hline SITCP15 & Solyc01g008230.2 & & GQ496333 \\
\hline SITCP16 & Solyc03g116320.2 & & GQ496334 \\
\hline SITCP17 & Solyc06g070900.2 & & GQ496335 \\
\hline SITCP18 & Solyc02g068200.1 & & GQ496336 \\
\hline SITCP19 & Solyc09g008030.1 & & GQ496337 \\
\hline SITCP2O & Solyc08g080150.1 & & GQ496338 \\
\hline SITCP21 & Solyc03g006800.1 & & GQ496339 \\
\hline SITCP22 & Solyc04g006980.1 & SITCP1 (fragment) & GQ496342 \\
\hline SITCP23 & Solyc05g007420.1 & & GQ496340 \\
\hline SITCP24 & Solyc08g048390.1 & & GQ496341 \\
\hline SITCP25 & Solyc05g009900.1 & & \\
\hline SITCP26 & Solyc03g045030.1 & & \\
\hline SITCP27 & Solyc02g094290.1 & & \\
\hline SITCP28 & Solyc02g065800.1 & & \\
\hline SITCP29 & Solyc08g048370.2 & & \\
\hline SITCP30 & Solyc10g008780.1 & & \\
\hline
\end{tabular}

showing no synteny with other genomes at all (not shown). Similarly, there is an almost perfect copy (3 mismatches in 529 nucleotides) of SITCP28, which is also associated with transposon-like sequences.

Phylogenetic analysis of the extended TCP domains of the predicted proteins following alignment together with the 24 known Arabidopsis TCP proteins is shown in Figure 1. The phylogenetic comparison with the Arabidopsis TCP proteins showed that conservation between proteins of the two species is usually low. Higher similarity of proteins within the same species indicates that gene duplications have occurred after the split between the two lineages. It also suggests that the higher number of genes in tomato, compared to Arabidopsis, is the result of more gene duplication events in tomato or of higher frequency 


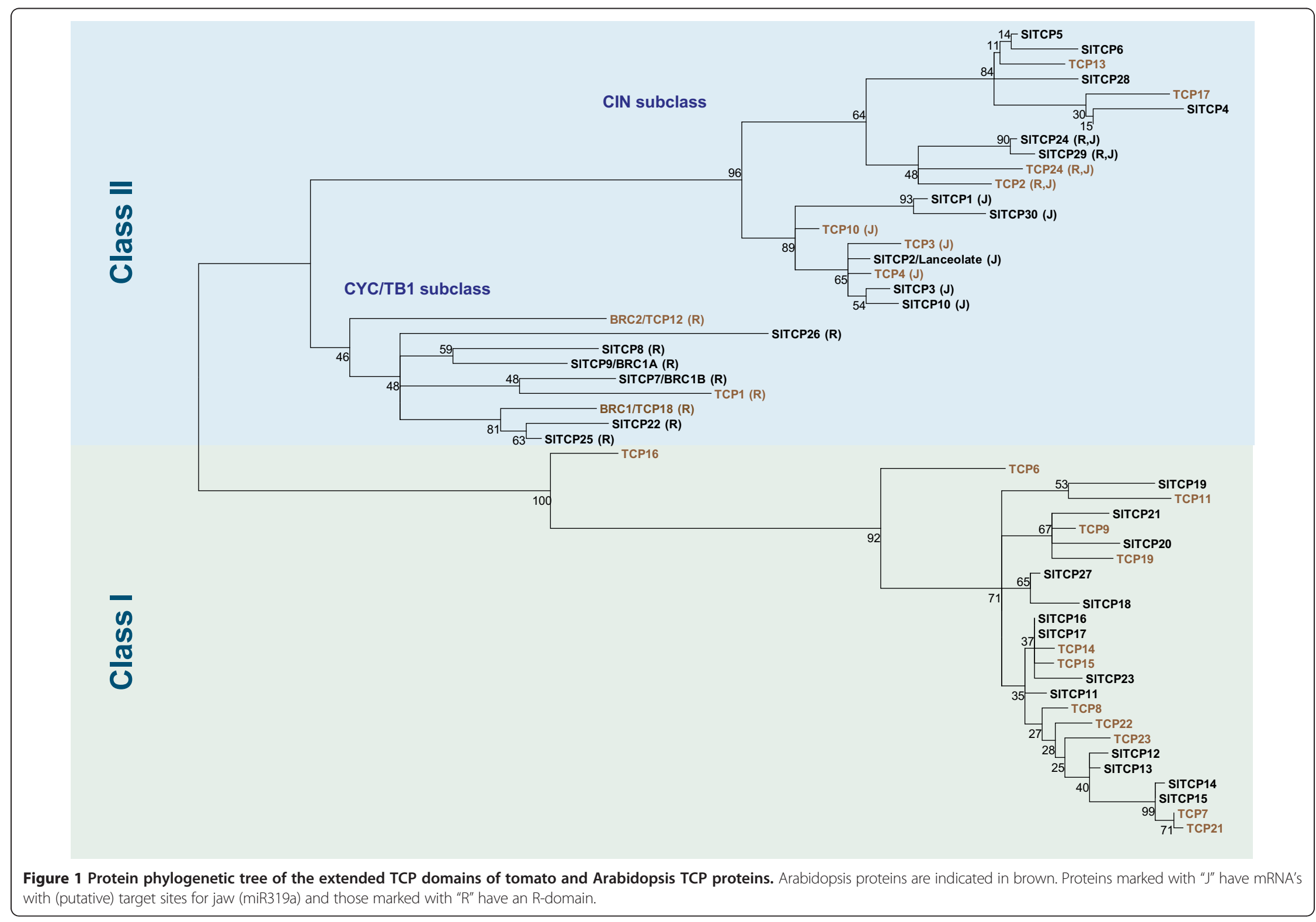




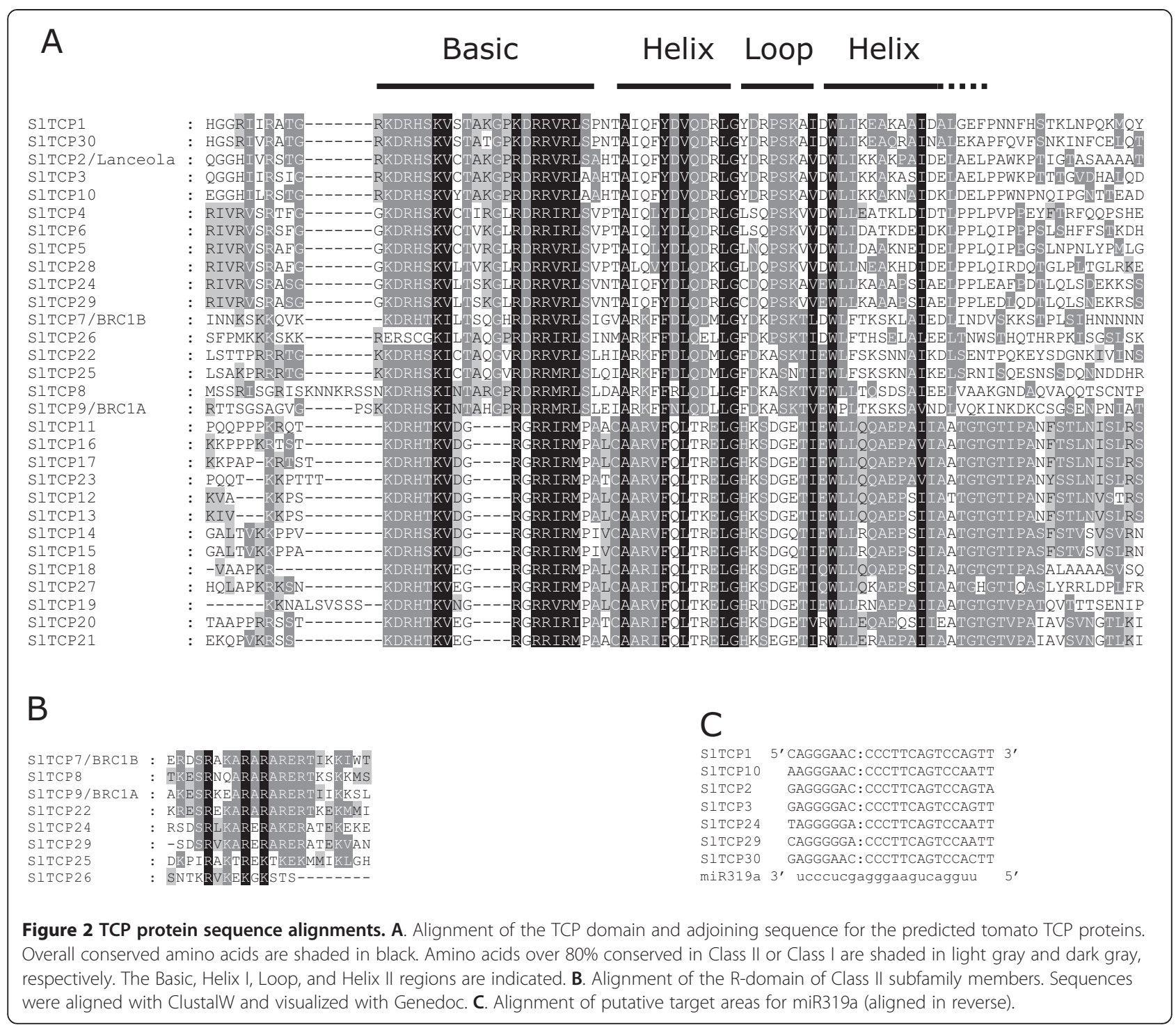

of retaining copies after duplication. Only the Arabidopsis AtTCP16 stands out as not having a close homolog in tomato.

The phylogenetic analysis showed that sequence conservation outside the approximately 60 amino acid TCP domain was low and sequence length on both sides of the TCP domain varied greatly, resulting in proteins ranging from 113 (TCP27) to 409 amino acids. The smallest predicted protein, SITCP27, is probably truncated by a frame shift mutation since sequence homology with Arabidopsis TCP20 extends well beyond the stop codon. The low overall conservation resulted in relatively low bootstrap values, indicating poor reliability of some of the branches. Analysis of the phylogenetic tree as well as of the alignment of the TCP domains (Figure 2A) showed that tomato TCP proteins can be divided into two subfamilies, as for all species so far. The CYC/TB1 or class II subfamily has, as reported earlier, an extended basic region, while class I subfamily members have extended homology C-terminal from the TCP domain, and both subfamilies have internally conserved, but distinct loop region sequences [1]. The phylogenetic tree also supported the Arabidopsis and rice earlier described division of class II proteins in two further subfamilies [33]. According to this division, class IIa or CYC/TB1 contains the tomato genes BRC1B (SlTCP7), SlTCP8, BRC1A (SlTCP9), SlTCP22, SlTCP25, and SlTCP26. From Figure 2A it is evident that SITCP26 lacks the conserved $\mathrm{N}$-terminal part of the basic region, which suggests that this protein may not be able to bind DNA.

Class IIb or CIN-TCPs, so named after their homology with Antirrhinum CINCINATTA, is a group of 8 TCP proteins in Arabidopsis involved in leaf growth regulation (AtTCP2, -3, -4, -5,-10,-13,-17, and -24) 
$[15,16]$. Tomato has 11 proteins in this homology group, among which the earlier identified LANCEOLATE, SITCP1 to SlTCP6, SlTCP10, -24, -28, -29 and -30 (Figure 1). The tree topology, particularly for class II, was further supported by analysis of synteny. Inspection of synteny in the Plant Genome Duplication Database (PGDD) found synteny between genomic regions for all tomato and Arabidopsis genes in the CYC/TB1-subclass, as well as for the genes from the subclade containing Lanceolate and the subclade containing tomato TCP4, $-5,-6$, and -28 . This suggests that members from these subclades originated from ancient whole genome or segmental duplications in a common ancestor of Arabidopsis and tomato. Tomato TCP24 and -29 do not show any synteny in the PGDD, whereas their closest Arabidopsis homologs do have extensive synteny with various species. Since these genes are arranged as a tandem inverted repeat on chromosome 8 , they may be the result of a lineagespecific rearrangement that is absent in Arabidopsis.

As reported earlier for Arabidopsis TCP1, TCP2, TCP12, TCP18, and TCP24, a subset of the closest tomato homologs of these Arabidopsis TCP proteins, BRC1B (SlTCP7), SITCP8, BRC1A (SITCP9), SITCP22, SlTCP24, and SITCP29 contain the so-called R-domain C-terminal of the TCP domain [33]. R domain-like sequences are also present in SITCP25 and SITCP26, but there they are less conserved (Figure 2B).

In Arabidopsis, 5 of the class IIb members are posttranscriptionally regulated by miRNA319 (AtTCP2, 3, 4, 10 , and 24) $[15,16,18]$. The closest tomato homologs of these Arabidopsis genes are the three new genes, SlTCP1, SlTCP29, and SlTCP30, and the earlier identified SITCP10, LANCEOLATE (SITCP2), SITCP3 and SlTCP24, respectively [15]), all having a putative binding site for $m i R 319 a$. This suggests that regulation of leaf development by a redundant set of miRNA-regulated homologous TCP genes occurs in tomato. SlTCP2, $-3,-10,-24$, and -30 have considerable expression, although not exclusively, in tomato leaves. Figure $2 \mathrm{C}$ shows the alignment of the target sites of these genes with the miR319a sequence. In a previously published degradome study, the transcripts of all putative miR319 targets identified here, with exception of SITCP30, were shown to be actually cleaved in tomato fruits [34].

\section{Expression analysis of the tomato TCP genes}

In order to predict possible functions as well as to identify probable functional redundancy through overlapping expression patterns for the tomato TCP genes, we determined expression levels of all 30 genes by quantitative RT-PCR. We used mRNA isolated from: tomato seedlings, leaves, roots, flowers at anthesis, flowers at 2 days post anthesis (DPA), immature green fruit at two sizes $(5 \mathrm{~mm}$ diameter and $18 \mathrm{~mm}$ diameter, respectively), mature green fruit, breaker stage fruit, turning stage fruit, and red ripe fruit. All expression levels (in this order) as related to the expression of the $\beta$-actin gene are shown in numerical order in Figure 3. From Figure 3 it is apparent that the expression levels in different organs vary widely between the tomato TCP genes, as well as between different organs for individual TCP genes. The representatives of class II TCPs have high expression mostly in flowers at anthesis, 2 DPA and leaves. In addition to the already mentioned organs, SlTCP25 shows relatively high expression in seedlings and lower in developing fruits.

We have identified 13 proteins as member of the class I group. Many class I genes seem more widely and less specifically expressed, such as in leaves, flowers, and early stages of developing fruits (Figure 3). SITCP11 and TCP27 appear to lack organ specificity, while SlTCP12 and SITCP18 are the only genes with high expression during ripening, with SITCP12 particularly rising at and beyond the mature green stage. Most class II genes are not expressed in fruits beyond the flower 2 DPA stage. SlTCP15 is relatively highly expressed up to the $5 \mathrm{~mm}$ fruit stage, after which the expression drops and comes back during ripening, with highest expression in the turning stage. The expression in tomato fruit is of particular interest since this is the first example of extensive characterization of TCP gene expression in a fleshy fruit species. SlTCP27 is regulated during ripening; however its expression is low in all tissues. SITCP12 and SITCP18 are particularly interesting because of their expression during fruit ripening. The expression pattern of SITCP12 is strikingly complementary to that of its closest homolog SlTCP13, possibly pointing to an example of neofunctionalisation after gene duplication through divergence of expression patterns. Opposite to the upward regulation of SlTCP12 during ripening, SITCP13, $-14,-15$ and -19 show high expression up to the $5 \mathrm{~mm}$ fruit stage, followed by a sharp drop and SlTCP15 being expressed again during ripening. The regulation of SITCP12, -15 and -18 during the onset of ripening may have functional significance that may not apply in non-fleshy Arabidopsis siliques.

\section{Interactions between tomato TCP proteins}

TCP proteins tend to form homodimers or heterodimers with other TCP proteins, and dimerization may be required for their DNA-binding activity and hence for their biological activity. We have determined dimer formation between 24 cloned tomato SITCP proteins in a yeast 2hybrid assay. Open reading frames were cloned as translational fusions with the yeast GAL4 transcription factor binding- (BD-) or activation- (AD-) domain and all combinations were tested in a matrix set-up. Results are represented schematically in Figure 4, where the proteins are arranged according to their phylogenetic 


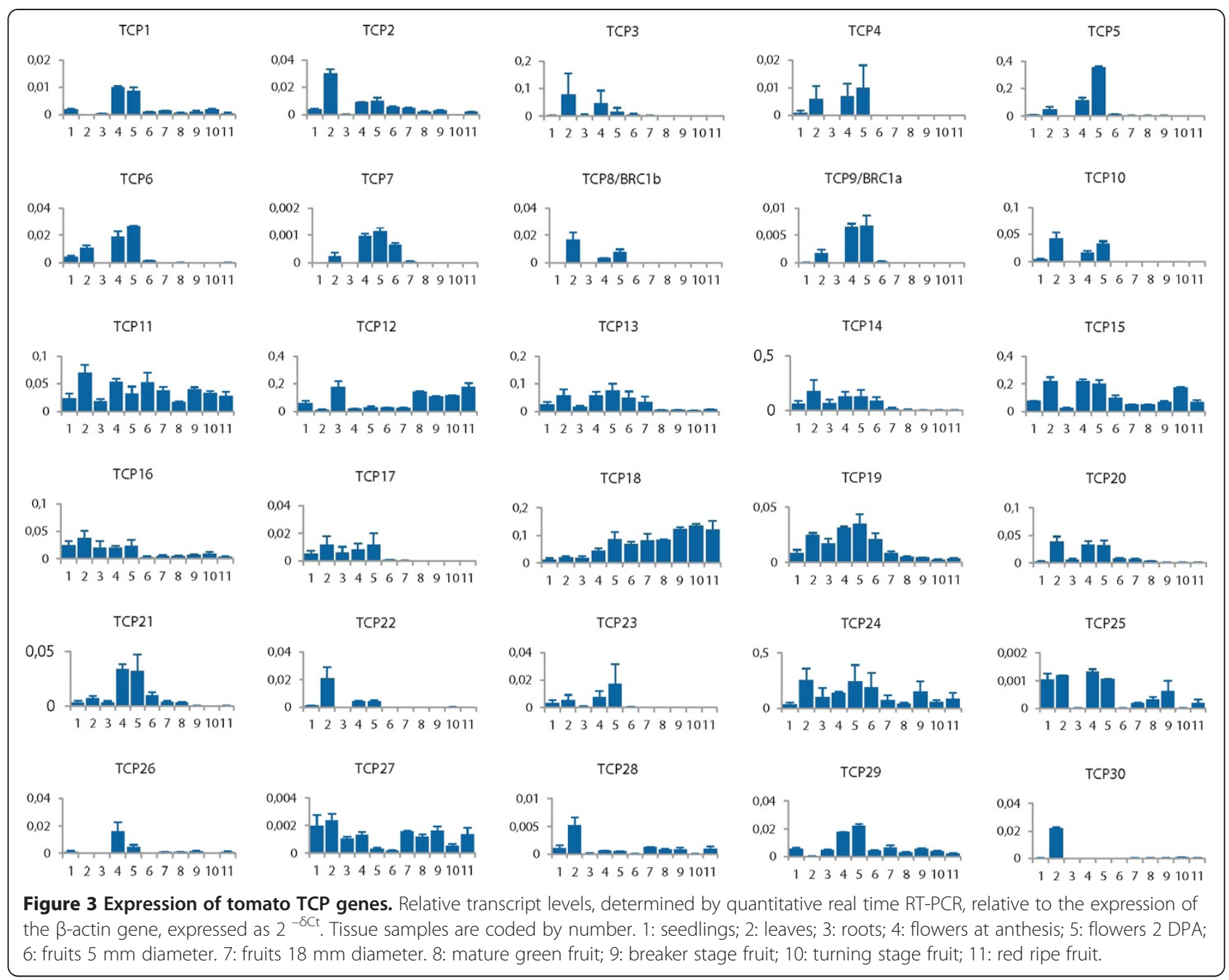

relatedness, and interaction scoring tables can be found in Additional file 3: Table S2. Of the 24 BD-fusion proteins tested, 5 had autoactivation activity in yeast (highlighted with asterisk in Figure 4) on both selection media, while four showed autoactivation only on -LTH medium. With the exception of SITCP12, these were found to be all class II TCP transcription factors, an overrepresentation that was also observed for Arabidopsis TCP transcription factors [7]. Thus, interactions could not be scored for the corresponding BD clones (empty rows in Figure 4) or could be scored only on -LTA medium. Altogether we observed 92 interactions, with a few exceptions on both selective media. Of these, in 34 (17 pairs) the partners interacted in both $\mathrm{BD} / \mathrm{AD}$-orientations, including 6 homodimer formations. The latter number may be an underestimation because homodimer formation could not be tested in the autoactivating family members. The summary of the interaction results shows that tomato TCP proteins form both homo- and heterodimers, in the latter case preferentially with proteins of the same class (77 interactions) as was previously noted for Arabidopsis [35], although a few (15) inter-class interactions were detected. SITCP12, which shows autoactivating activity on one medium, has only SITCP6 and SITCP21 overlapping and five different interactions compared to its nearest homolog SITCP13, which is not autoactivating. Together with the different expression patterns, this points to functional divergence after the gene duplication. Again similar to the Arabidopsis TCP proteins, more interactions were found for class I proteins than were found for Class II proteins (42 versus 33), although also here the number of interactions for Class II proteins may be underestimated because of the autoactivating members. The interactions obtained by a comprehensive yeast two-hybrid screen of the tomato TCP transcription factors, has not yet been reported to such extent for TCP members from other species than Arabidopsis. These yeast 2-hybrid interactions of the tomato TCP genes confirmed earlier observations for 


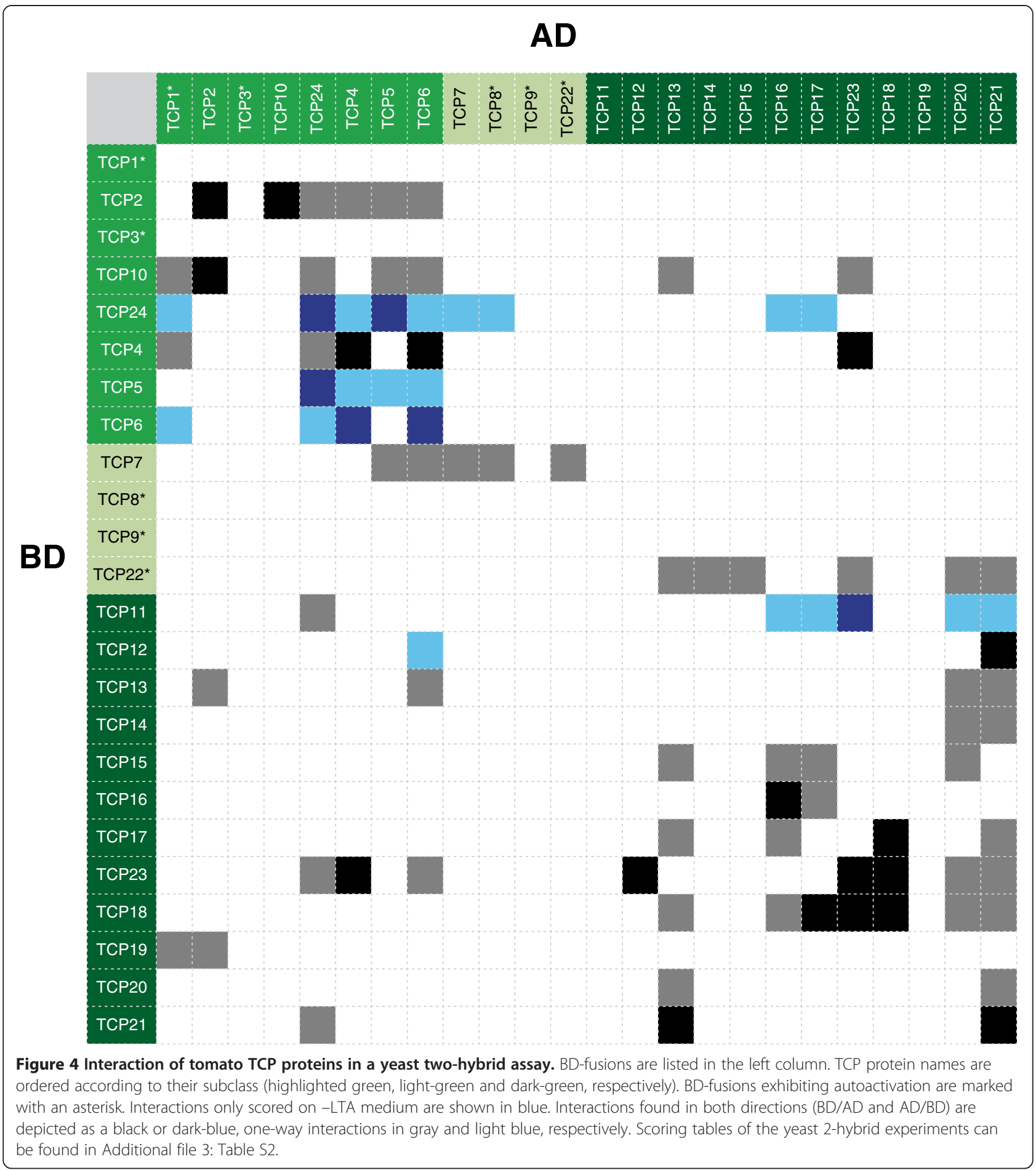

rice $[23,25]$, showing that TCP proteins form homodimers, and heterodimers particularly with proteins within the same class. The combination of expression analysis and dimerization properties may in the future help to identify TCP protein pairs that function together and to explain observed functional redundancies in case of overlapping interaction maps.
The expression of the tomato TCP12, TCP15 and TCP18 genes is affected by mutations in major ripening regulatory genes

We demonstrated that SlTCP12, and -18 show differential expression during fruit ripening, with SITCP12 highly expressed in the ripening stages and SITCP18 being increasingly expressed from early stages gradually to 


\section{$\begin{array}{ll}\text { A) SIAP2a knock-down } & \text { B) } \mathrm{Cnr}\end{array}$}
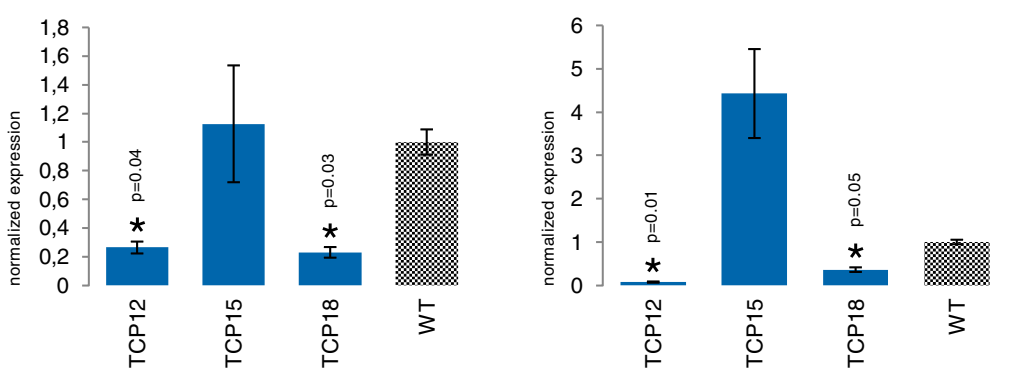

C) rin

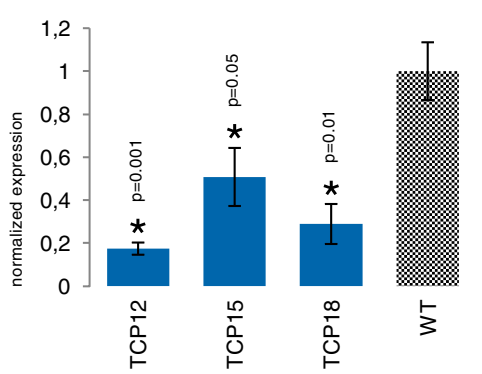

D) nor

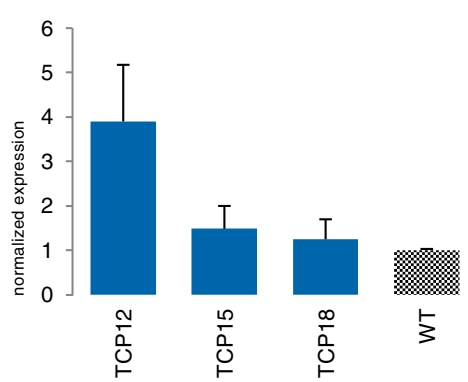

Figure 5 Normalized expression of SITCP12, SITCP15 and SITCP18 in tomato fruit at Breaker +7 stage. Expression values are normalized for each gene related to wild type, expressed as $2^{-\delta \delta C t}$. A. In the ripening defective transgenic plants with RNAi-suppressed SIAP2a. T-test p-values: 0.04 (SITCP12), 0.81 (SITCP15), and 0.03 (SITCP18). B. In the ripening defective mutant Cnr, p-values: 0.01 (SITCP12), 0.19 (SITCP15), and 0.05 (SITCP18). C. In the ripening defective mutant rin, p-values: 0.001 (SITCP12), 0.05 (SITCP15), and 0.01 (SITCP18). D. In the ripening defective mutant nor, p-values: 0.3 (SITCP12), 0.5 (SITCP15), and 0.7 (SITCP18). Asterisks indicate significant differences ( $p \leq 0.05)$.

Table 2 Transcription factors binding promoter fragments in a yeast one-hybrid assay

\begin{tabular}{lccc}
\hline & \multicolumn{3}{c}{ promoter fragment } \\
\cline { 2 - 4 } Tomato protein & SITCP12 & SITCP15 & SITCP18 \\
\hline SIAP2a & - & - & - \\
RIN & + & + & + \\
CNR & - & - & + \\
SITCP19 & - & + & - \\
SITCP11 & + & - & - \\
SITCP20 & - & + & - \\
SITCP6 & + & - & - \\
SITCP9 & + & + & + \\
SITCP2 & - & - & + \\
SITCP23 & - & + & - \\
SITCP18 & - & + & - \\
SITCP22 & - & + & - \\
SITCP16 & - & + & - \\
SITCP1 & + & + &
\end{tabular}

Representing the binding of: SIAP2a, RIN and CNR, as well as the tomato TCP proteins to the promoter elements of SITCP12, -15 and -18 . "-" represents no binding, " + " represents binding. red ripe fruits. SlTCP15 expression is high in flowers at anthesis, 2 DPA and $5 \mathrm{~mm}$ fruit, which are stages associated with a high mitotic index rate [36] and then regulated again during ripening, peaking in the breaker stage. The expression patterns of these TCPs suggested that they might be positively or negatively regulated by ripening, and thus directly or indirectly by some of the known major regulators of this process. We determined the expression of these TCP genes by qRT-PCR analysis performed in fruits of the $\mathrm{Br}+7$ (7 days after the breaker) stage of the tomato ripening-defective mutants Cnr (Colorless non-ripening) [37], rin (ripening-Inhibitor) [38], nor [39] and in transgenic SlAP2a knock-down plants [40]. As expected, the transcript levels of SITCP12, SlTCP15 and SITCP18 are regulated in several of the ripening mutants (Figure 5A, 5B, 5C). SlTCP12, associated with ripening, is significantly and positively regulated by SlAP2a, CNR and RIN (Figure 5A, 5B, 5C), while the expression of SITCP15, associated with early fruit development, is not statistically significantly regulated by $S l A P 2 a$ and $C N R$ (Figure 5A,5B). RIN positively and significantly regulates SITCP15 (Figure 5C). SlTCP18, which has expression in all stages of fruit development, with a high increase during ripening, is significantly down regulated in $S I A P 2 a$ RNAi, Cnr and rin (Figure 5A, 5B, 5C). The three TCPs are 
not significantly regulated in the nor mutant (Figure 5D). The positive regulation of SITCP12 and SITCP18 by CNR, $S l A P 2 a$ and RIN, indicates that they might be positively associated with ripening. Additionally, RIN positively regulates SlTCP15, associating it also with the ripening process.

\section{Ripening-related tomato transcription factor proteins bind SITCP12,-15 and -18 promoter elements}

The altered expression of SITCP12, -15 and -18 in the ripening mutants and $S I A P 2 a$ transgenic knockdown plants indicates that they are regulated by key ripening regulatory genes, but does not show if this regulation is direct. In order to find out if these transcription factors could be directly interacting with the promoters of SlTCP12, SlTCP15 and SlTCP18, we used yeast-one-hybrid assays to identify transcription factors binding to the promoter fragments of the ripening related SITCP genes. The promoter elements used were upstream of the transcription start sites and were as follows: 568-bp promoter fragment for SlTCP12, 500-bp fragment of SlTCP15 and 473-bp fragment of SITCP18. In this assay we detected interaction between the ripening regulator RIN and the promoters of SlTCP12, -15 and -18 as well as between CNR and the promoter of TCP18 (Table 2). This data strongly suggests that the regulation of these TCPs by RIN and CNR is direct.

In the cases where regulation of SITCP expression by ripening regulatory transcription factors was detected, but no binding to the target promoter was observed, several other scenarios are possible. The length of the promoter fragments used in the assay is limited and may not have comprised all putative transcription factor binding sites. Alternatively, regulation of expression may be indirect via regulation of expression of other transcription factors that do bind the target promoter.

\section{Arabidopsis transcription factors binding the promoter fragments of SITCP12, SITCP15 and SITCP18}

To identify more potential regulators of tomato SlTCP12, SlTCP15 and SITCP18, we performed a yeast one-hybrid assay with transcription factors fused to the GAL4 activation domain. Since there is no large collection of cloned tomato transcription factors, instead we opted for initially testing the available REGIA collection of Arabidopsis transcription factors, consisting of 1397 cloned transcription factor gene open reading frames [41]. All transcription factors interacting with one or more of the promoter fragments are listed in Additional file 4: Table S3. These results demonstrate that the SITCP12, -15 and -18 promoter fragments interact with 115, 99 and 86 different Arabidopsis transcription factors, respectively. Nine Arabidopsis transcription factors bind the promoters of all three SITCP genes (Additional file 4: Table S3). One of those is the ethylene-responsive gene AtDEAR1
(DREB and EAR motif protein 1), also named CEJ1 (COOPERATIVELY REGULATED BY ETHYLENE AND JASMONATE 1). In Arabidopsis, DEAR1 expression is induced by pathogen infection [42].

The common interactions for SITCP15 and SlTCP18 promoter fragments, in addition to the AtDEAR1/CEJ1 protein, are nine. SITCP12 and SITCP15 share additional 11 common interactions. SlTCP12 and SlTCP18 have 32 common interactions, among which one encodes a member of the DREB subfamily A-2 of ERF/AP2 transcription factor family, another ethylene responsive gene. The fact that both SITCP12 and SITCP18 have more common interactions compared to SITCP12 and SlTCP15 or SlTCP18 and SlTCP15, may be due to their expression overlap during ripening.

This experiment demonstrates that there are a lot of Arabidopsis transcription factors binding the promoter elements of SITCP12, -15 and -18 . Whether the same trend can be observed with tomato transcription transcription factors may be further investigated. In the absence of a comprehensive library of cloned tomatos TFs, we speculate that the same would be observed for tomato TFs. Recent studies in Arabidopsis show that many binding sites can be occupied by transcription factors, in a dynamic manner without them necessarily being active regulators of the bound promoter [43]. We performed Gene Onthology (GO-) term enrichment analysis for transcription factors binding to each of the three SITCP promoters versus the tested 1379 Arabidopsis transcription factors from the REGIA collection. The results did not show significant enrichment per GO category, suggesting that these TCPs may be involved in or regulated by many different processes.

Some of the Arabidopsis proteins binding SlTCP12 promoter fragment are transcription factors known to be part of the ethylene signal transduction pathway. These include an ERF/AP2 transcription factor gene encoding for RELATED TO AP2 3 (RAP2.3), other genes from the ERF (ETHYLENE RESPONSE TRANSCRIPTION FACTOR) family, and ETHYLENE-INSENSITIVE3-LIKE 2 (EIL2). Several AP2/ERF proteins also bind SlTCP12, SlTCP15 and SITCP18 independently (Additional file 4: Table S3). The regulation of SITCP genes by ethylene responsive genes may indicate an indirect mechanism of ethylenedependant expression of these SITCPs during ripening.

\section{Tomato transcription factors binding to the SITCP12, -15} and -18 promoter elements

Using the observed interactions with Arabidopsis transcription factors as a lead to candidate regulators from tomato, we selected some of the strongest interacting proteins and identified their closest tomato homologs by Blast homology searches. These were subsequently cloned and used in a yeast one-hybrid assay as described above. The tomato genes used are listed in Table 3 next to their 
Table 3 Binding of Arabidopsis and tomato transcription factors to TCP promoter fragments

\begin{tabular}{|c|c|c|c|c|c|c|c|c|c|}
\hline \multirow[b]{2}{*}{ Arabidopsis protein } & \multirow[b]{2}{*}{ AGI } & \multicolumn{3}{|c|}{ Promoter fragment } & \multirow[b]{2}{*}{$\begin{array}{l}\text { Corresponding } \\
\text { tomato homolog }\end{array}$} & \multirow[b]{2}{*}{$\begin{array}{l}\text { Tomato } \\
\text { protein name }\end{array}$} & \multicolumn{3}{|c|}{ Promoter fragment } \\
\hline & & SITCP12 & SITCP15 & $\overline{\text { SITCP18 }}$ & & & SITCP12 & SITCP15 & $\overline{\text { SITCP18 }}$ \\
\hline DEAR1/CEJ1 & AT3G50260 & + & + & + & Solyc04g078640.1 & & + & - & + \\
\hline WOX13 & AT4G35550 & - & + & + & Solyc02g082670.2 & & - & - & - \\
\hline KNAT5 & AT4G32040 & + & + & - & Solyc07g007120.2 & LeT12 & + & - & - \\
\hline KNAT4 & AT5G11060 & + & - & + & Solyc07g007120.2 & LeT12 & + & - & - \\
\hline SCL18/LAS & AT1G55580 & + & - & + & Solyc07g066250.1 & LS & - & - & - \\
\hline ettin/ARF3 & AT2G33860 & + & - & - & Solyc02g077560.2 & ARF3 & - & - & - \\
\hline AlF3 & AT3G17100 & + & - & - & Solyc01g058670.2 & & - & - & - \\
\hline RVE1 & AT5G17300 & - & + & - & Solyc02g036370.2 & & + & - & - \\
\hline RAV1/EDF4 & AT1G13260 & - & - & + & Solyc05g009790.1 & & + & - & - \\
\hline NAC13 & AT1G32870 & - & - & + & Solyc12g056790.1 & & - & - & - \\
\hline SVP & AT2G22540 & - & - & + & Solyc11g010570.1 & JOINTLESS & + & - & + \\
\hline bHLH115 & AT1G51070 & - & - & + & Solyc07g064040.2 & & - & - & + \\
\hline AGL18 & AT3G57390 & - & - & + & Solyc01g087980.2 & & - & - & - \\
\hline
\end{tabular}

Yeast one-hybrid results, representing the binding of the selected Arabidopsis transcription factor proteins and the corresponding tomato putative orthologs to the tomato TCP12, -15 , and -18 promoter elements independently. "-" represents no binding, "+" represents binding.

Arabidopsis homologs, together with their interactions with the tomato TCP promoters.

The Arabidopsis DEAR1, also named CEJ1 binds the promoter fragments of SITCP12, -15 and -18 (Additional file 4: Table S3) and its identified tomato homolog Solyc04g078640.1 interacts with the SITCP12 and SlTCP18 promoters (Table 3). LeT12 is the tomato homolog of the Arabidopsis KNAT4 and KNAT5, which bind to the SlTCP12 and -15 and SlTCP12 and -18 promoter fragments, respectively. The tomato homolog LeT12 however binds only to the SITCP12 promoter (Table 3). LeT12 [44] is a class II KNOX gene (Solyc07 g007120.2), expressed in all tissues, but has high expression in green fruits and leaves [45].

Arabidopsis SVP (SHORT VEGETATIVE PHASE) binds the SITCP18 promoter fragment. The tomato homolog of SVP, JOINTLESS (J) binds to both SlTCP12 and SlTCP18 promoter fragments (Table 3). In Arabidopsis, SVP controls flowering time and maintains the meristematic activity during the early floral meristem stages [46], while in tomato $J$ regulates pedicel abscission zone formation and maintenance of the inflorescence meristem [47].

\section{TCP transcription factors bind the promoters of SITCP12, SITCP15 and SITCP18}

The protein-DNA interaction assay performed with the Arabidopsis TF collection and the tomato promoter fragments show that there are Arabidopsis TCPs binding the promoter fragments of tomato TCP12, -15 and -18 (Additional file 4: Table S3). AtTCP6 and -13 bind the SlTCP18 promoter, while AtTCP1, $-3,-4,-8$, and -19 bind the SITCP15 promoter. The SITCP12 promoter did not show any binding activity with Arabidopsis TCP transcription factors.

In the yeast one-hybrid assay we also screened for promoter-binding activity of the tomato TCP proteins. This experiment revealed that the binding of SITCP12, -15 and -18 by the Arabidopsis TCP transcription factors are seen also with most of their tomato SITCP homologs. SlTCP1, -6, -9, which are class II TCP TFs, bind the class I SITCP12 promoter (Table 2). Expression of these proteins peaks in flowers and early fruit development, in contrast to SlTCP12, which peaks from mature green fruit onwards and in roots. These complementary expression patterns suggest that binding of the class II TCPs inhibits SlTCP12 expression. Interestingly, SITCP11, a class I TCP regulated in all tissues and closest homolog of SlTCP12, also binds to the SITCP12 promoter (Table 2).

SlTCPs binding the SlTCP15 promoter are SITCP1, -9, and -22 , which are class II TCPs, strongly expressed in flowers at anthesis and 2 DPA, and in leaves (Table 2). Class I SITCPs also bind the SITCP15 promoter. Among them is SITCP18, whose expression increases during ripening (Table 2). SITCP15 shows a different trend of regulation, being regulated by more representatives of its own class. Generally, the proteins from class I and II TCPs binding SlTCP15 are expressed in flowers, early fruit development and leaves, which fits well with the expression of SlTCP15. Therefore, we can hypothesize that this binding leads to activating the expression of SITCP15. The regulation of SITCP15 by SITCP18 may be linked to ripening, because both genes have higher expression during breaker and turning fruit stages. 


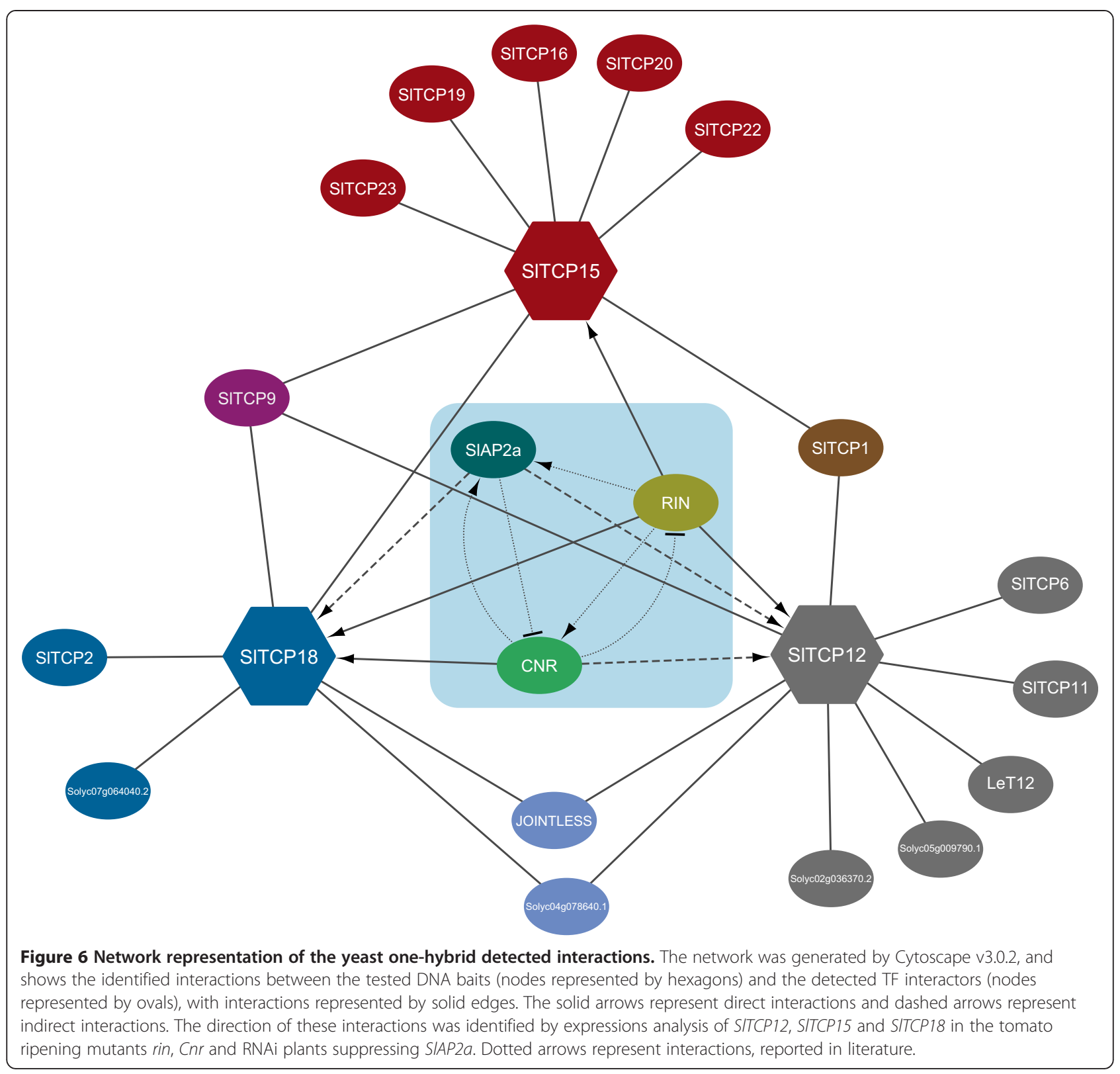

SlTCP18 promoter is bound only by class I SITCPs, which expression patterns are complementary to that of SlTCP18 (Table 2). This suggests that these class I SITCPs may regulate $S I T C P 18$ in a negative way.

The protein-DNA interaction between TCP transcription factors and SITCP12,-15 and -18 promoter elements suggest that TCPs regulate the expression of other members of their own family. This regulatory network is visualized in Figure 6. It also suggests that SITCPs from class II regulate SITCPs from class I, and SITCPs from class I regulate SlTCPs from the same class. This cross-regulation among SITCP genes suggests that class II SITCPs may act as repressors of class I, but on the other hand, class I may activate genes from their own class, as this may be the case with SlTCP15. Thus, the tomato TCP genes are likely part of an interrelated regulatory network, as has already been described for TCP genes in Arabidopsis [7]. Since these regulatory interactions were inferred from yeast one-hybrid assay results, which are prone to producing false positives, further in vivo and/or in planta experiments are needed to confirm these interactions.

\section{Conclusions}

In conclusion, we have identified the 30 members of the tomato TCP transcription factor gene family. Tomato genes closely related to Arabidopsis TCP genes, have similar expression patterns, which suggests conserved functions. Additionally, the tomato TCP proteins form 
homodimers and heterodimers particularly with SITCPs from the same class (Figure 4). This trend was reported before also in other species, like Arabidopsis [35] and rice [25]. SITCP19 is an exception, because it is a class I SlTCP, dimerizing only with class II SlTCPs (SlTCP1 and SlTCP2). Interestingly, the tomato TCP12, TCP15 and TCP18 genes show differential expression patterns during fleshy fruit development and ripening. Expression studies show that SITCP12, -15 and -18 are positively regulated by the ripening regulators RIN, CNR and SlAP2a, which are among the promoter binding proteins of these TCP genes (Figure 6). These data shows that the SlTCP12, -15 and -18 genes are directly or indirectly controlled by these ripening regulators and might play a role in tomato fruit ripening. Furthermore, we show that SITCP proteins can bind to the promoter sequences of other SITCP genes (Figure 6), suggesting that they could coordinately or competitively regulate their expression. Our promoter and protein-protein interaction studies suggest that SITCP12, -15 and -18 , may be involved in a variety of other functions, in addition to fruit ripening. Further research analyzing the phenotypes of knockout or knock down of SITCP12, -15 or -18 will reveal more information about the function of these genes.

\section{Methods}

\section{Plant Material}

Solanum lycopersicum 'Moneymaker' was used as source for plant material. Seedlings were grown on agar culture for 21 days. For the collection of roots, seedlings were grown along a Whattman filter paper and fed with 0.5 MS medium for 28 days. For the other samples, plants were grown in the greenhouse and the following tissues or organs were harvested: fully expanded leaves, flowers at anthesis, flowers two days post anthesis, developing fruits with $5 \mathrm{~mm}$ diameter, developing fruits with $18 \mathrm{~mm}$ diameter, mature green fruits, fruits at breaker stage, fruits at turning stage and fruit at red ripe stage. Plant materials were frozen in liquid nitrogen and ground with IKA A11basic (Staufen, Germany). The material was stored at $-80^{\circ} \mathrm{C}$ until further use.

\section{TCP sequence identification and cloning}

In order to identify tomato genes putatively encoding TCP transcription factors, the Solanaceae Genomics Network (SGN)-Unigene database (for ESTs) as well as the BAC sequences deposited by the International Tomato Sequencing Project were searched using the TBLASTN algorithm with Arabidopsis TCP proteins or TCP domains as query sequence. For thus identified unigenes, EST clones, when available, were obtained from the Boyce Thompson Institute for Plant Research at Cornell University (Ithaca, New York) and resequenced to confirm identity and to establish the presence or absence of 5' and 3' ends of an open reading frame. Where either one or both open reading frame ends were not present or where no EST was available, mRNA sequence was extended by 5 ' and/or 3' RACE, as was done for three partial cDNA sequences deposited earlier in GenBank (AAO45726, AAO45727, AAO45728 [31]). For three putative TCP gene sequences found in BAC sequences only, the genomic DNA sequence was taken as template for the design of RACE primers. For this purpose, RNA was isolated of tomato fruit pericarp of 7 developmental stages ( 7 days after pollination to red ripe). mRNA was isolated using the RNAeasy Plant MiniKit (50) (QIAGEN). All RNA's were mixed together in equal amounts. 5'and 3' RACE-ready cDNA libraries were made from RNA using the Clontech SMART RACE cDNA amplification kit (Westburg B.V., Leusden, the Netherlands). Full-length open reading frames were amplified by PCR using ESTs (when containing the entire orf), 5' and 3' RACE clones, or genomic DNA. Genomic DNA was isolated from leaves using an earlier described method [48]. All sequencing and open reading frame amplification templates, as well as primers used for RACE PCR and open reading frame PCR are listed in Additional file 2: Table S4 and S5. Open reading frames were amplified using primers with a 5' CACC extension, and purified using the QIAquick PCR Purification Kit or purified from gel using the QIAquick gel extraction kit (Qiagen) and ligated between GATEWAY attL1 and attL2 sites in pENTR/D-TOPO using an Invitrogen pENTR ${ }^{\mathrm{m}}$ Directional $\mathrm{TOPO}^{\circ}$ Cloning Kits (www.lifetechnologies. com), producing GATEWAY Entry vectors. Ligated products were transformed into E. coli DH5 $\alpha$ competent cells by electroporation. All entry clones were checked by sequencing analysis (BigDye sequencing kit, Applied Biosystems). (DYEnamic ET Terminator (Dett) Cycle Sequencing Kit from Amersham Biosciences, GE Healthcare). The messenger RNA sequences of the first identified 24 SlTCPs have been deposited in GenBank under accession numbers listed in Table 1.

\section{Phylogenetic analyses}

Tomato TCP protein sequences were compared with all 24 A. thaliana TCP proteins. Multiple sequence alignment was performed with Muscle [49] as implemented in MEGA v5.10 [50]. Phylogenetic reconstruction was obtained by the NJ (neighbor-joining) method [51] using the Jones-Taylor-Thornton (JTT) substitution model with Gamma-distributed rates (5 categories) among sites together with bootstrap analysis using 500 replicates.

\section{Gene expression analysis}

Extraction of total RNA was performed with the use of TriPure reagent (Roche Diagnostics, Indianapolis, USA) according to the manufacturer's protocol. DNAase 
treatment was performed with DNAase I (Invitrogen, Breda, the Netherlands) according to the protocol. RNeasy columns (Qiagen, Venlo, the Netherlands) were used to purify the RNA. Quantitative and qualitative concentration measurements were performed using Nanodrop ND-1000 Spectrophotometer (NanoDrop Technologies, Delaware, USA).

For determination of transcript concentrations, quantitative real time RT-PCR was performed in two biological replicates. Briefly, $1 \mu \mathrm{g}$ of total RNA was used for cDNA synthesis using the TaqMan Reverse Transcription Reagents kit (Roche Molecular Systems, Branchburg, USA). Primers were designed using Beacon Designer (Biosoft International, Palo Alto, USA) and purchased from Biolegio (Nijmegen, The Netherlands). Real-Time PCR was performed in a MyIQ Single-Color Real-Time PCR Detection System (Bio-Rad, Veenendaal, The Netherlands) using the following program: 3 min denaturation at $94^{\circ} \mathrm{C}, 40$ cycles of $15 \mathrm{sec}$ at $94^{\circ} \mathrm{C}$ and $30 \mathrm{sec}$ at $60^{\circ} \mathrm{C}$, followed by a melting curve gradient to analyze the specificity of the primer pairs for a particular gene. No Template-Controls served as blanks and $\beta$-Actin was used as reference gene based on least variation observed in the 11 tomato tissues. For the ripening mutants, $\mathrm{Cnr}$, rin, nor, and the knock down SlAP2 $a$, RNA from Breaker +7 was isolated using an InviTrap ${ }^{\circ}$ Spin Plant RNA Mini Kit (www.invitek.de). The RNA quality was checked on gel and cDNA was synthesised using TaqMan ${ }^{\circledR}$ Reverse Transcription Reagents Invitrogen $^{\mathrm{TM}}$ ) with $1 \mu \mathrm{g}$ of RNA. The qRT-PCR was performed on BIORAD iQ5 using SYBR-green fluorescence dye. The program used was as described before for the TCPs qRT-PCR.

Sequences of the primer pairs used are listed in Additional file 2: Table S6. $\mathrm{C}_{\mathrm{t}}$-values of the 11 samples were measured in duplo and averaged, followed by calculation of the relative gene expression using the $2^{-\delta C t}$ method for the expression of SITCP transcripts in the different tomato organs and the $2^{-\delta \delta C t}$ method [52] for the expression of SITCPs in the ripening mutants. Analysis of the reaction efficiency was performed with the LinRegPCR program [53].

\section{Yeast two-hybrid assays}

All TCP ORFs were recombined from the entry clone into the pBDGAL4 bait vector (pDEST ${ }^{\mathrm{Tm}} 32$, Invitrogen) and pADGAL4 prey vector ( $\mathrm{pDEST}^{\mathrm{T}} 22$, Invitrogen). The bait vectors were transformed into yeast strain PJ69-4 $\alpha$ $(M A T \alpha)$ and all prey vectors into strain PJ69-4a (MATa [54]) and selected on SD plates lacking Leu and Trp, respectively. Subsequently, overnight cultures were grown $\left(30^{\circ} \mathrm{C}, 300 \mathrm{rpm}\right)$ from single colonies of each transformant in selective SD medium and systematically mated with each other by spotting $5 \mu \mathrm{L}$ droplets of the liquid cultures on top of each other on SD complete plates (Nunc Omnitray;
VWR International, Amsterdam, the Netherlands) containing all the essential amino acids. In addition, some negative control combinations were spotted, for which water was used instead of either a bait or prey culture. Subsequently, the plates were incubated at $30^{\circ} \mathrm{C}$ for $16 \mathrm{~h}$, and afterwards the yeast was transferred to SD plates lacking both Leu and Trp to select for diploid yeast containing both plasmids. After $2 \mathrm{~d}$ of growth at $30^{\circ} \mathrm{C}$, the yeast was transferred to four different selection plates containing SD medium lacking Leu, Trp, and Ade (-LTA) and SD lacking Leu, Trp, and His (-LTH), supplemented with 5, 10, or $15 \mathrm{mM}$ 3-amino-1,2,4-triazole (3-AT), respectively. Bait clones were tested for autoactivation in the absence of an interacting prey protein by plating on SD lacking Leu and His supplemented with increasing concentrations of 3-AT. For subsequent interaction experiments only growth on selective media (if any), where no growth of autoactivating clones occurred, was scored. These plates were incubated at $20^{\circ} \mathrm{C}$ and scored for growth of yeast and hence protein-protein interaction events after $5 \mathrm{~d}$. The screening was performed in triplicate. In case of autoactivation for one of the two proteins, just four data points were obtained for the specific combination. The mating efficiency appeared to be $100 \%$, and where water was used for mating, either instead of a bait culture or instead of a prey culture, no growth was obtained on medium selecting for the presence of the two plasmids or on the media selecting for interactions. This shows that no cross-contamination occurred as a result of the procedure that followed. A combination was scored as a true interaction when it resulted in growth for at least one of the two selection markers (Adenine or Histidine) in at least three out of four experiments. Combinations that grew only on one selective medium were marked as such in the presentation of the results.

\section{Yeast one-hybrid assay}

DNA-protein interactions between Arabidopsis transcription factors and the single tomato TCP12, TCP15 and TCP18 proteins were identified and characterized using yeast-one hybrid assay, which was based on Clontech's Matchmaker Gold Yeast One-Hybrid (Y1H) System (http://www.clontech.com). This system uses the antibiotic Aureobasidin A resistance as a reporter. PJ69-4A yeast strain was used for the transcription factor baits and PJ69- $\alpha$ for the TCP12, -15 and -18 promoter reporter constructs. The single promoter fragments were cloned into the pAbAi reporter vector, which was made Gateway compatible. Primers used for the promoter elements cloning can be seen in Additional file 2: Table S7. The reporter construct of TCP12 consists of 568-bp (region SL1.03sc00008:1785048..1785615), TCP15 - of 500-bp (region SL2.31sc04133:30626689..30627956) and TCP18 of 473-bp (region SL1.03sc01076:4199969..4200441). 
For each of the promoter fragments, an autoactivation test was performed with Aureobasidin A concentrations ranging from 0 to $500 \mathrm{ng} / \mathrm{ml}$. The $\mathrm{Y} 1 \mathrm{H}$ screenings were done at concentration of $75 \mathrm{ng} / \mathrm{ml}$ Aureobasidin A for each reporter, which was the lowest background activation detected. The yeast clones were grown and selected for the presence of the plasmid for 2 to $3 \mathrm{~d}$ on selective medium at $30^{\circ} \mathrm{C}$. The transcription factors bait and the TCPs reporter clones were mated on SD complete medium overnight and then transferred onto bait/reporter selection medium for 3 days. The media-selected mated yeasts were transferred to $100 \mu \mathrm{L}$ of sterile MilliQ water and $5-\mu \mathrm{L}$ droplets were spotted onto aureobasidincontaining plates. Plates were incubated at $20^{\circ} \mathrm{C}$ and scored after 5 to 7 days.

The transcription factors (TFs) library (The REGIA TF ORF Library) used for the yeast one-hybrid screen contains a set of Arabidopsis thaliana transcription factor open reading frames (ORFs) [41]. After identification of the protein - DNA binding, the Arabidopsis TFs, which were found to strongly bind the promoter of the studied tomato TCP proteins, were characterized as candidate genes. The selection of the tomato candidate TFs was based on their closest Arabidopsis homolog, characterized by protein nucleotide blast search in the database of SOL Genomics Network. Some of the tomato candidate genes, which had expression during fruit development and ripening (based on EST and Unigene expression data) and the tomato homologs of the strongest bound Arabidopsis TFs were selected and cloned (Additional file 4: table S3).

\section{Cloning of the tomato candidate TFs used in the yeast one-hybrid screen}

The ORFs of the single tomato candidate TFs were amplified and independently cloned in $\mathrm{pCR}^{\mathrm{Tm}} 8 / \mathrm{GW} / \mathrm{TOPO}$ vector (Invitrogen). All entry clones were controlled by sequencing analysis, then recombined into the pADGAL4 vector ( $\mathrm{pDEST}^{\mathrm{TM}} 22$, Invitrogen) and transformed into PJ69-4A yeast strain [55]. In summary, 40 tomato open reading frames were cloned (Additional file 2: Tables S5 and S8). The yeast one-hybrid experiment was performed in triplicate with the tomato TFs.

\section{Cytoscape - network data integration, analysis and visualization}

Cytoscape v3.0.2 [56] was used for generating network of the yeast-one hybrid interactions and for integrating the expression data of SITCP12, -15 and -18 in the ripening mutants $C n r$, rin and the SlAP2a knockdown plants. Default settings were used with nested network style.

BiNGO 2.8 plugin for Cytoscape [57] was used for GO term enrichment analysis. In the search for overrepresentation we used the standard settings: Benjamini-Hochberg FDR, significance level of 0.05 .

\section{Availability of supporting data}

The data sets supporting the results of this article are included within the article and its additional files (additional figures and tables).

\section{Additional files}

Additional file 1: Figure S1. Chromosomal location of the tomato TCP genes. "1a-d", "19a:, and "28a" depict the extra copies of the respective genes in the tomato reference genome.

Additional file 2: Table S1. Additional (partial) copies of SITCP1, -19, and -28 found in the tomato reference genome. Table S4. Tomato TCP genes and TCP-like sequences, corresponding SGN-Unigenes, (re) sequenced EST clones, and RACE experiments performed to obtain full-length CDNAs. Table S5. Sequences of primers used for amplification of open reading frames. Table S6. Sequences of primers used for quantitative RT-PCR. Table S7. Primers used for amplification of TCP12, TCP15 and TCP18 promoters. Table S8. Primers used for the amplification of the ORF of tomato candidate genes.

Additional file 3: Table S2. Scoring tables for the yeast two-hybrid experiments. Every experiment was performed in triplicate and interactions were scored as positive if growth occurred on both selection media in at least two of the three experiments.

Additional file 4: Table S3. Yeast one-hybrid results, representing the binding of the Arabidopsis transcription factor proteins and the cloned corresponding tomato homologous proteins to the tomato TCP12, -15 , and -18 promoter elements independently. "-" represents no binding, "+" represents binding.

\section{Competing interests}

The authors declare that they have no competing interests.

\section{Authors' contributions}

Authors, who have made substantial contributions to conception, design of experiments, acquisition of data, analysis and interpretation of data: VP, RD, $A B, R K$. Authors who have contributed to performing experiments: VP, ML, $M B, J B$. Authors who have been involved in drafting the manuscript: VP and RM. Authors who have revised it critically: VP, RM, RK, GA. Authors who have given final approval of the version to be published: all. Authors who agree to be accountable for all aspects of the work in ensuring that questions related to the accuracy or integrity of any part of the work are appropriately investigated and resolved: all.

\section{Acknowledgments}

We thank Lukasz Patyk, Froukje van der Wal and Richard Immink for assistance with the yeast two-hybrid and yeast one-hybrid assays, and the latter as well as Selahattin Danisman for useful discussions. Mrs Ruth White (Boyce Thompson Institute, Ithaca, NY) is gratefully acknowledged for sending us tomato EST clones. This work was partially funded by European Commission $6^{\text {th }}$ Framework programme project "High Quality Solanaceous crops for consumers, processors and producers by exploration of natural biodiversity" (EU-SOL; contract nr. PL 016214-2), by the Dutch Ministry of Agriculture, Nature and Food Quality and the Dutch Organization for Scientific Research NWO-ALW project nr. 828.08.005.

\section{Author details}

${ }^{1}$ Laboratory of Molecular Biology, Department of Plant Sciences,

Wageningen-UR, PO Box 386, 6700 AJ Wageningen, the Netherlands. ${ }^{2}$ Plant Research International, P.O. Box 619, 6700 AP Wageningen, the Netherlands.

${ }^{3}$ Molecular Plant Physiology, Utrecht University, Padualaan 8, $3584 \mathrm{CH}$ Utrecht, the Netherlands.

Received: 21 February 2014 Accepted: 22 May 2014

Published: 6 June 2014 


\section{References}

1. Cubas P, Lauter N, Doebley J, Coen E: The TCP domain: a motif found in proteins regulating plant growth and development. Plant J 1999, 18(2):215-222.

2. Martin-Trillo M, Cubas P: TCP genes: a family snapshot ten years later. Trends Plant Sci 2010, 15(1):31-39.

3. Uberti Manassero NG, Viola IL, Welchen E, Gonzalez DH: TCP transcription factors: Architectures of plant form. Biomolecular Concepts 2013, 4(2):111-127.

4. Tatematsu K, Nakabayashi K, Kamiya Y, Nambara E: Transcription factor AtTCP14 regulates embryonic growth potential during seed germination in Arabidopsis thaliana. Plant J 2008, 53(1):42-52.

5. Kieffer M, Master V, Waites R, Davies B: TCP14 and TCP15 affect internode length and leaf shape in Arabidopsis. Plant J 2011, 68(1):147-158.

6. Herve C, Dabos P, Bardet C, Jauneau A, Auriac MC, Ramboer A, Lacout F, Tremousaygue D: In vivo interference with AtTCP20 function induces severe plant growth alterations and deregulates the expression of many genes important for development. Plant Physiol 2009, 149(3):1462-1477.

7. Danisman $S$, van der Wal F, Dhondt $S$, Waites R, de Folter S, Bimbo A, van Dijk AD, Muino JM, Cutri L, Dornelas MC, Angenent GC, Immink GH: Arabidopsis class I and class II TCP transcription factors regulate jasmonic acid metabolism and leaf development antagonistically. Plant Physiol 2012, 159(4):1511-1523.

8. Takeda T, Amano K, Ohto MA, Nakamura K, Sato S, Kato T, Tabata S, Ueguchi C: RNA interference of the Arabidopsis putative transcription factor TCP16 gene results in abortion of early pollen development. Plant Mol Biol 2006, 61(1-2):165-177.

9. Luo D, Carpenter R, Vincent C, Copsey L, Coen E: Origin of floral asymmetry in Antirrhinum. Nature 1996, 383(6603):794-799.

10. Guo Z, Fujioka S, Blancaflor EB, Miao S, Gou X, Li J: TCP1 modulates brassinosteroid biosynthesis by regulating the expression of the key biosynthetic gene DWARF4 in arabidopsis thaliana. Plant Cell 2010, 22(4):1161-1173.

11. Doebley J, Stec A, Hubbard L: The evolution of apical dominance in maize. Nature 1997, 386(6624):485-488.

12. Aguilar-Martínez JA, Poza-Carrión C, Cubas P: Arabidopsis BRANCHED1 acts as an integrator of branching signals within axillary buds. Plant Cell 2007, 19(2):458-472.

13. Martin-Trillo M, Grandio EG, Serra F, Marcel F, Rodriguez-Buey ML, Schmitz G, Theres K, Bendahmane A, Dopazo H, Cubas P: Role of tomato BRANCHED1like genes in the control of shoot branching. Plant J 2011, 67(4):701-714.

14. Crawford BCW, Nath U, Carpenter R, Coen ES: CINCINNATA controls both cell differentiation and growth in petal lobes and leaves of antirrhinum. Plant Physiol 2004, 135(1):244-253.

15. Ori N, Cohen AR, Etzioni A, Brand A, Yanai O, Shleizer S, Menda N, Amsellem Z, Efroni I, Pekker I, Alvarez JP, Blum E, Zamir D, Eshed Y: Regulation of LANCEOLATE by miR319 is required for compound-leaf development in tomato. Nat Genet 2007, 39(6):787-791.

16. Palatnik JF, Allen E, Wu XL, Schommer C, Schwab R, Carrington JC, Weigel D: Control of leaf morphogenesis by microRNAs. Nature 2003, 425 (6955):257-263.

17. Nath U, Crawford BC, Carpenter R, Coen E: Genetic control of surface curvature. Science 2003, 299(5611):1404-1407.

18. Palatnik JF, Wollmann H, Schommer C, Schwab R, Boisbouvier J, Rodriguez R, Warthmann N, Allen E, Dezulian T, Huson D, Carrington JC, Weigel D: Sequence and expression differences underlie functional specialization of Arabidopsis microRNAs miR159 and miR319. Dev Cell 2007, 13(1):115-125.

19. Nag A, King S, Jack T: miR319a targeting of TCP4 is critical for petal growth and development in Arabidopsis. Proc Natl Acad Sci U S A 2009, 106(52):22534-22539.

20. Schommer C, Palatnik JF, Aggarwal P, Chételat A, Cubas P, Farmer EE, Nath $U$, Weigel D: Control of jasmonate biosynthesis and senescence by miR319 targets. PLoS Biol 2008, 6(9):e230.

21. Pruneda-Paz JL: A functional genomics approach reveals $\mathrm{CHE}$ as a component of the Arabidopsis circadian clock (vol 325, pg 1481, 2009). Science 2009, 326(5951):366-366.

22. Koyama T, Furutani M, Tasaka M, Ohme-Takagi M: TCP transcription factors control the morphology of shoot lateral organs via negative regulation of the expression of boundary-specific genes in Arabidopsis. Plant Cell 2007, 19(2):473-484.

23. Kosugi S, Ohashi Y: PCF1 and PCF2 specifically bind to cis elements in the rice proliferating cell nuclear antigen gene. Plant Cell 1997, 9(9):1607-1619.
24. Aggarwal P, Das Gupta M, Joseph AP, Chatterjee N, Srinivasan N, Nath U: Identification of specific DNA binding residues in the TCP family of transcription factors in Arabidopsis. Plant Cell 2010, 22(4):1174-1189.

25. Kosugi S, Ohashi Y: DNA binding and dimerization specificity and potential targets for the TCP protein family. Plant J 2002, 30(3):337-348.

26. Tremousaygue D, Garnier L, Bardet C, Dabos P, Herve C, Lescure B: Internal telomeric repeats and 'TCP domain' protein-binding sites co-operate to regulate gene expression in Arabidopsis thaliana cycling cells. Plant $J$ 2003, 33(6):957-966.

27. Li C, Potuschak T, Colon-Carmona A, Gutierrez RA, Doerner P: Arabidopsis TCP20 links regulation of growth and cell division control pathways. Proc Natl Acad Sci U S A 2005, 102(36):12978-12983.

28. Vrebalov J, Ruezinsky D, Padmanabhan V, White R, Medrano D, Drake R, Schuch W, Giovannoni J: A MADS-box gene necessary for fruit ripening at the tomato ripening-inhibitor (rin) locus. Science 2002, 296(5566):343-346.

29. Manning K, Tor M, Poole M, Hong Y, Thompson AJ, King GJ, Giovannoni JJ, Seymour GB: A naturally occurring epigenetic mutation in a gene encoding an SBP-box transcription factor inhibits tomato fruit ripening. Nat Genet 2006, 38(8):948-952.

30. Chung MY, Vrebalov J, Alba R, Lee J, McQuinn R, Chung JD, Klein P, Giovannoni J: A tomato (Solanum lycopersicum) APETALA2/ERF gene, SIAP2a, is a negative regulator of fruit ripening. Plant J 2010, 64(6):936-947.

31. Reeves PA, Olmstead RG: Evolution of the TCP gene family in Asteridae: cladistic and network approaches to understanding regulatory gene family diversification and its impact on morphological evolution. Mol Biol Evol 2003, 20(12):1997-2009.

32. Lee $T H$, Tang $H$, Wang $X$, Paterson AH: PGDD: a database of gene and genome duplication in plants. Nucleic Acids Res 2012, 41(D1):D1152-D1158.

33. Yao X, Ma H, Wang J, Zhang DB: Genome-wide comparative analysis and expression pattern of TCP gene families in Arabidopsis thaliana and Oryza sativa. J Integr Plant Biol 2007, 49(6):885-897.

34. Karlova R, van Haarst JC, Maliepaard C, van de Geest H, Bovy AG, Lammers M, Angenent GC, de Maagd RA: Identification of microRNA targets in tomato fruit development using high-throughput sequencing and degradome analysis. J Exp Bot 2013, 64(7):1863-1878.

35. Danisman S, Van Dijk ADJ, Bimbo A, Van Der Wal F, Hennig L, De Folter S, Angenent GC, Immink RGH: Analysis of functional redundancies within the Arabidopsis TCP transcription factor family. J Exp Bot 2013, 64(18):5673-5685.

36. Srivastava A, Handa AK: Hormonal regulation of tomato fruit development: A molecular perspective. J Plant Growth Regul 2005, 24(2):67-82.

37. Thompson AJ, Tor M, Barry CS, Vrebalov J, Orfila C, Jarvis MC, Giovannoni JJ, Grierson D, Seymour GB: Molecular and genetic characterization of a novel pleiotropic tomato-ripening mutant. Plant Physiol 1999, 120(2):383-390.

38. Robinson RW, Tomes ML: Ripening inhibitor: a gene with multiple effect on ripening. Tomato Genet Coop 1968, 18:36-37.

39. Tigchelaar EC, Tomes ML, Kerr EA, Barman RJ: A new fruit ripening mutant, non-ripening (nor). Rep Tomato Genet Coop 1973, 23:33-34.

40. Karlova R, Rosin FM, Busscher-Lange J, Parapunova V, Do PT, Fernie AR, Frase PD, Baxter C, Angenent GC, de Maagd RA: Transcriptome and metabolite profiling show that APETALA2a is a major regulator of tomato fruit ripening. Plant Cell 2011, 23(3):923-941.

41. Castrillo G, Turck F, Leveugle M, Lecharny A, Carbonero P, Coupland G, Paz-Ares J, Onate-Sanchez L: Speeding cis-trans regulation discovery by phylogenomic analyses coupled with screenings of an arrayed library of Arabidopsis transcription factors. PLoS One 2011, 6(6):e21524.

42. Tsutsui T, Kato W, Asada Y, Sako K, Sato T, Sonoda Y, Kidokoro S, YamaguchiShinozaki K, Tamaoki M, Arakawa K, Ichikawa T, Nakazawa M, Seki M, Shinozaki K, Matsui M, lkeda A, Yamaguchi J: DEAR1, a transcriptional repressor of DREB protein that mediates plant defense and freezing stress responses in Arabidopsis. J Plant Res 2009, 122(6):633-643

43. Pajoro A, Madrigal P, Muiño JM, Matus JT, Jin J, Mecchia MA, Debernardi JM, Palatnik JF, Balazadeh S, Arif M, Maoiléidigh DS O, Wellmer F, Krajewski P. Riechmann JL, Angenent GC, Kaufmann K: Dynamics of chromatin accessibility and gene regulation by MADS-domain transcription factors in flower development. Genome Biol 2014, 15(3):R41.

44. Janssen BJ, Williams A, Chen JJ, Mathern J, Hake S, Sinha N: Isolation and characterization of two knotted-like homeobox genes from tomato. Plant Mol Biol 1998, 36(3):417-425.

45. Ozaki S, Ogata Y, Suda K, Kurabayashi A, Suzuki T, Yamamoto N, lijima Y, Tsugane T, Fujii T, Konishi C, Inai S, Bunsupa S, Yamazaki M, Shibata D, Aoki K: 
Coexpression analysis of tomato genes and experimental verification of coordinated expression of genes found in a functionally enriched coexpression module. DNA Res 2010, 17(2):105-116.

46. Lee JH, Yoo SJ, Park SH, Hwang I, Lee JS, Ahn JH: Role of SVP in the control of flowering time by ambient temperature in Arabidopsis. Genes Dev 2007, 21(4):397-402.

47. Mao L, Begum D, Chuang HW, Budiman MA, Szymkowiak EJ, Irish EE, Wing RA: JOINTLESS is a MADS-box gene controlling tomato flower abscission zone development. Nature 2000, 406(6798):910-913.

48. Fulton TM, Chunwongse J, Tanksley SD: Microprep protocol for extraction of DNA from tomato and other herbaceous plants. Plant Mol Biol Rep 1995, 13(3):207-209.

49. Edgar RC: MUSCLE: multiple sequence alignment with high accuracy and high throughput. Nucleic Acids Res 2004, 32(5):1792-1797.

50. Tamura K, Peterson D, Peterson N, Stecher G, Nei M, Kumar S: MEGA5: molecular evolutionary genetics analysis using maximum likelihood, evolutionary distance, and maximum parsimony methods. Mol Biol Evol 2011, 28(10):2731-2739.

51. Saitou N, Nei M: The neighbor-joining method: a new method for reconstructing phylogenetic trees. Mol Biol Evol 1987, 4(4):406-425.

52. Livak KJ, Schmittgen TD: Analysis of relative gene expression data using real-time quantitative PCR and the $2^{\text {-ddCT }}$ method. Methods 2001, 25(4):402-408.

53. Ramakers C, Ruijter JM, Deprez RH, Moorman AF: Assumption-free analysis of quantitative real-time polymerase chain reaction (PCR) data. Neurosci Lett 2003, 339(1):62-66.

54. James P, Halladay J, Craig EA: Genomic libraries and a host strain designed for highly efficient two-hybrid selection in yeast. Genetics 1996, 144(4):1425-1436.

55. de Folter S, Immink RG, Kieffer M, Parenicova L, Henz SR, Weigel D, Busscher M, Kooiker M, Colombo L, Kater MM, Davies B, Angenent GC: Comprehensive interaction map of the Arabidopsis MADS Box transcription factors. Plant Cell 2005, 17(5):1424-1433.

56. Cline MS, Smoot M, Cerami E, Kuchinsky A, Landys N, Workman C, Christmas R, Avila-Campilo I, Creech M, Gross B, Hanspers K, Isserlin R, Kelley R, Killcoyne S, Lotia S, Maere S, Morris J, Ono K, Pavlovic V, Pico AR, Vailaya A, Wang PL, Adler A, Conklin BR, Hood L, Kuiper M, Sander C, Schmulevich I, Schwikowski B, Warner GJ, et al: Integration of biological networks and gene expression data using Cytoscape. Nat Protoc 2007, 2(10):2366-2382.

57. Saito R, Smoot ME, Ono K, Ruscheinski J, Wang PL, Lotia S, Pico AR, Bader GD, Ideker T: A travel guide to Cytoscape plugins. Nat Methods 2012, 9(11):1069-1076.

doi:10.1186/1471-2229-14-157

Cite this article as: Parapunova et al.: Identification, cloning and characterization of the tomato TCP transcription factor family. BMC Plant Biology 2014 14:157.

\section{Submit your next manuscript to BioMed Central and take full advantage of:}

- Convenient online submission

- Thorough peer review

- No space constraints or color figure charges

- Immediate publication on acceptance

- Inclusion in PubMed, CAS, Scopus and Google Scholar

- Research which is freely available for redistribution 